\title{
Allelic variations between vaccine strains and circulating strains in pxtP of Bordetella pertussis in Iran
}

\author{
Sadeghpour Heravi F, Nikbin VS, Shahcheraghi F*
}

Pertussis reference laboratory, Department of Bactriology, Pasteur Institute of Iran, Tehran, Iran.

\begin{abstract}
Introduction: Despite high level of vaccination against pertussis, whooping cough has re-emerged as a health threat, especially in infants. This could be related to expansion of Bordetella pertussis with novel alleles for virulence factors including the pertussis toxin promoter, ptxP3. Compared to ptxp1 strains, ptxp3 strains produce more pertussis toxin which results in immune suppression and virulence. The main aim of this study was the determination of dominant alleles of the promoter region of the gene coding for pertussis toxin in B. pertussis isolates in Iran. Methods: In this project, we studied the allelic variations in ptxP3 .This factor was sequenced and analyzed in $35 \mathrm{~B}$. pertussis isolates from Iranian patients in 2011. Biochemical tests were performed to confirm the B. pertussis isolates. Ultimately, polymerase chain reactions and sequencing were done. Results: Our results showed that the predominant allele among the strains was ptxP3. One isolated strain (i.e. ptxP1) showed different results from the other strains. Also, B. pertussis 134 and 509 as common vaccine strains used in Iran were analyzed and they were identified to be ptxP1. Conclusion: Based on our results in recent years, the vaccine strains and the circulating strains do not share the same alleles which could be one of the causes of pertussis resurgence in the world. ptxP is a well-known toxin of B. pertussis which is responsible for binding to the host cell and the disruption of the cellular function. In particular, allelic variation in ptxP may play a role in adaption of $B$. pertussis.
\end{abstract}

KEYWORDS: Bordetella pertussis, virulence factors, polymorphism, vaccine.

\section{INTRODUCTION}

Bordetella pertussis is the etiologic agent of whooping cough, a respiratory disease which is sever in infants [1]. According to World Health Organization (WHO) in 2008, there were 15 million cases of pertussis which $95 \%$ were in the developing countries, ultimately $200^{\prime} 000$ children died from whooping cough on that time[2]. According to recent WHO reports, there are 50 million infected pertussis cases which could lead to 300 '000 deaths every year in the world [3]. Although pertussis is a vaccine-preventable disease [4], it has remained a health problem in many vaccinated populations [2]. In recent years, reports of the resurgence of pertussis and the pathogen adaption have been published in many countries with long histories of vaccination against pertussis such as the USA [5] and Australia [6]. Different causes have been proposed for the resurgence of pertussis such as: waning vaccine-induced immunity and pathogen adaptation [7]. Meanwhile, increased awareness and

*Corresponding Author: Fereshteh Shahcheraghi, Ph.D; Pertussis reference laboratory, Department of Bactriology, Pasteur Institute of Iran, Tehran, Iran.

Email: shahcheraghifereshteh@yahoo.com

Tel/Fax: (+98) 2166405535 improved diagnosis techniques have been accounted for increased pertussis notifications [8].

There are two types of pertussis vaccine, namely whole-cell (wP) and acellular (aP). wP has often been produced from killed circulating strains but some countries have acquired other sources [6]. aP is a new generation of the vaccine that contains 1-5 different components of B. pertussis[9]. The length of the protection from vaccination with aP vaccine and $\mathrm{wP}$ vaccine and the natural infection can vary. After the natural infection, the immunity wanes in 7-20 years while after the vaccination, this period becomes shorter, in range of 4-12 years. There is also evidence indicating that aP may give shorter even protection than wP [10]. Most European countries including Germany, Italy, Finland, Sweden and Luxembourg use aP vaccines. In Australia and France, aP vaccine is also used for the adolescents [8]. In Iran, pertussis vaccination has been introduced since 1950 s with high coverage by wP vaccines till now [11]. Despite utilization of aP in many developed and developing countries, the resurgence of pertussis has been reported in recent years $[12,13]$.

$B$. pertussis produces a wide range of virulence factors which have important roles in the infection and transmission of the disease including; pertussis toxin, tracheal cytotoxin, 
adenylatecyclase-hemolysin, filamentous hemagglutinin, fimbriae and pertactin [14]. Allelic variations have been found in the virulence associated genes encoding the pertussis toxin, such as pertactin, serotype 2 fimbriae, serotype 3 fimbriae and the promoter for pertussis toxin which is used in the acellular vaccines [14]. Many variations are reported in the pertussis toxin promoter region. The mutations in the promoter region of pertussis toxin occur at the sites where the activator of transcription BvgA binds (Fig. 1)[15, 16]. So far, 14 alleles with respect to the promoter region of pertussis toxin have been found [17-19]. Pertactin is an outer membrane protein which acts as an adhesion and to date, 13 alleles have been reported for it [5].

Large shifts have been observed in B. pertussis populations in the western countries. These shifts have been occurred after the introduction of the vaccination program, suggesting that they were probably vaccine-driven [20,21]. It has been proposed that the mismatches are mainly important in hosts with waning immunity that allow $B$. pertussis to re-infect earlier after the vaccination. The combination of waning immunity and the pathogen adaptation may be the most important reason for the re-emergence of pertussis [22]. The main aim of this study was to determine the ptxP alleles among the circulating $B$. pertussis strains, compared with the vaccine strains in Iran. Due to the lack of information on this issue in Iran, it would be important to study the antigenic variations in this promoter and other important virulence factors of $B$. pertussis in the Iranian isolates.

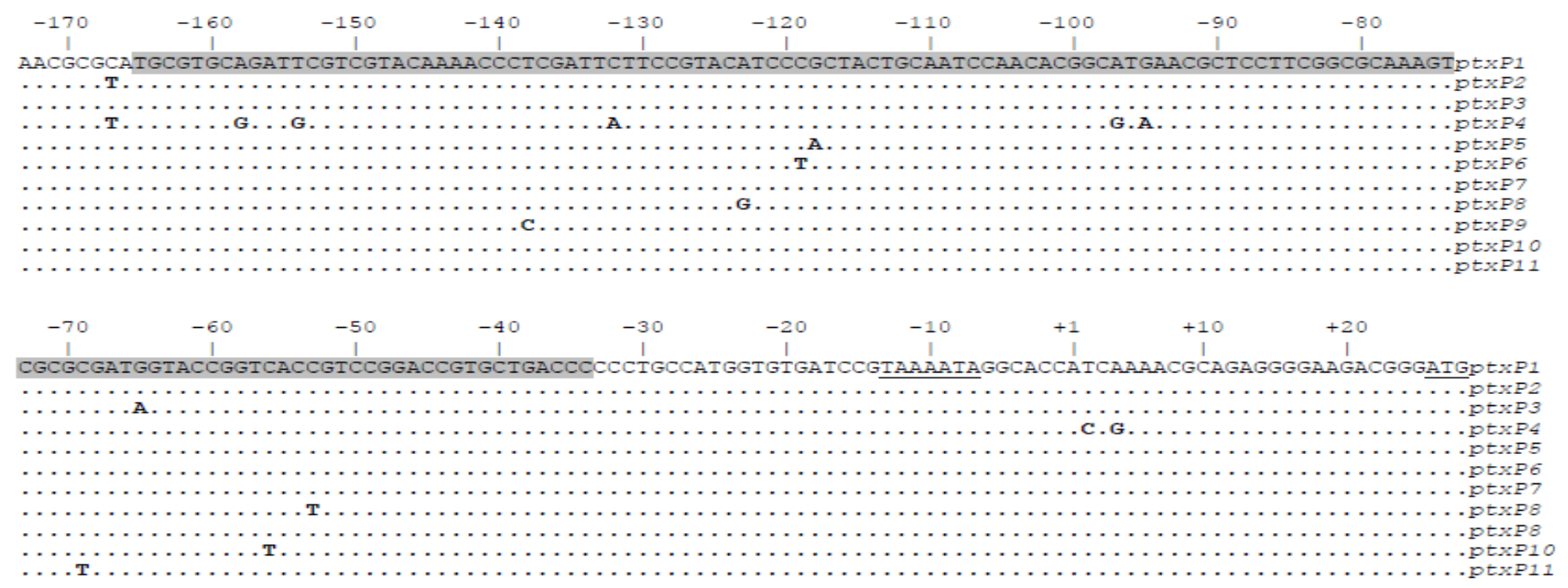

Fig. 1. Alleles of pertussis toxin promoter (ptxP) observed worldwide[15].

\section{MATERIALS and METHODS}

\section{Bacterial strains}

Nasopharyngeal Dacron swab samples (1301 in total) on Regan-Lowe transport medium were transported from different provinces of Iran to the Pertussis Reference Laboratory at the Pasteur Institute of Iran in 2011. These suspected specimens were cultured on Bordet-Gengou and Reagan-Lowe media (Difco, USA). All plates were incubated for 3-10 days at 35-36 ${ }^{\circ} \mathrm{C}$. Oxidase and catalase positive coccobacilli were selected for confirmation of $B$. pertussis using conventional biochemical tests (API 20E; Biomerieuxsa, France). Slide agglutination test for identification of $B$. pertussis as a confirmatory test with specific antiserum was also done (Difco, USA). B. pertussis 134 and 509 which are two strains presently used for vaccine production in Iran were analyzed as the vaccine strains.

\section{DNA extraction}

DNA extraction was performed by high pure PCR template preparation kit (Roche Diagnostics GmbH, Mannheim, Germany).

\section{PCR and DNA sequencing}

PCR temperature condition and primers used in this research are described in Table 1 . The ptxP gene was amplified in $25 \mu \mathrm{L}$ PCR mixture with $1 \mu \mathrm{M}$ of each primer. DNA sequencing was performed after amplification of the target genes. The sequencing process was carried out by Macrogen Company in South Korea.

Table 1. Primers and PCR program used in this study

\begin{tabular}{|c|c|c|c|c|c|}
\hline Gene & Primer & Sequence (5'-3') & PCR program & PCR product (bp) & Ref. \\
\hline \multirow{5}{*}{$p t x P$} & & & $15 \mathrm{~min} 95^{\circ} \mathrm{C}$ & & \\
& PF & AATCGTCCTGCTCAACCGCC & $15 \mathrm{~s} 95^{\circ} \mathrm{C}$ & 574 & {$[21]$} \\
& & & $30 \mathrm{~s}$ at $65^{\circ} \mathrm{C}$ & & \\
& PR & GGTATACGGTGGCGGGAGGA & 1 min at $72^{\circ} \mathrm{C}$ & & \\
& & & 30 cycles; $10 \mathrm{~min} 72^{\circ} \mathrm{C}$ & & \\
\hline
\end{tabular}




\section{RESULTS}

This study focused on polymorphism in ptxP. As a result, out of 1301 suspected specimens, 35 were positive by culture. In this study, we determined two different ptxP alleles among the specimens. The predominant allele in the clinical strains, except one case and the vaccine strains was identified ptxP3, and the different allele was ptxP1 (Fig. 2). Table 2 contains detailed information about the history of the clinical strains.
According to the specimens which were transported to Pertussis Reference Laboratory at the Pasteur Institute of Iran (Table 2), the infection affects mostly the children, less than 18 months old. For example in 2011, out of 35 positive samples, 27 cases were originated from children less than 18 months old while 17 cases were vaccinated at least by one dose of wP vaccine [23]. The different result among specimens is related to a one and a half month-old male infant that his nasopharyngeal Dacron swab was transported from Mashhad in Iran. Also there was not any dose of vaccination in history of this patient.

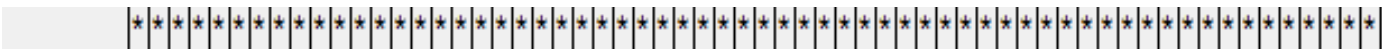 ptxp-1 AACGCGCATGCGIGCAGATICGICGTACAAAACCCTCGATICIICCCGIACAICCCECCIACIG ptxp-31 AaCGCGCATGCGTGCAGATICGTCGTACAAAACCCTCGATICTICCGTACAICCCGCTACTG}

Fig. 2. Alignment of $p t x P 1$ with strain number 31

Table 2. Distribution of pertussis in confirmed cases by age, isolated in 2011.

\begin{tabular}{|c|c|c|c|c|c|}
\hline Suspected sample & $\begin{array}{c}\text { No. of } \\
\text { isolates }\end{array}$ & Less than $\mathbf{2} \mathbf{~ m}$ & $\mathbf{2 - 1 8} \mathbf{~ m}$ & $\mathbf{1 8} \mathbf{~ m - 1 0} \mathbf{~}$ & $\mathbf{1 0} \mathbf{y}$ and older \\
\hline 1301 & 35 & 10 & 17 & 7 & 1 \\
\hline
\end{tabular}

Abbreviations: $\mathrm{m}=$ month, $\mathrm{y}=$ year

\section{DISCUSSION}

Pertussis, also known as whooping cough, is mainly caused by bacterium B. pertussis. Whooping cough is considered as a vaccine-preventable disease in the world. It is sever especially in infants who do not have a complete immune system to protect them against $B$. pertussis. Vaccination programs against pertussis were initiated in the 1940s-1960s in different countries around the world. Contrary to high vaccination coverage of pertussis in the developing as well as the industrialized countries, pertussis has reminded one of the top 10 causes of death in children worldwide [24].

In the 1990s, a resurgence of pertussis was observed in several countries with highly vaccinated populations [25]. According to a recent report from WHO, there are 50 million cases of pertussis disease annually [26].According to the Centers for Disease Control and Prevention (CDC), the incidence of pertussis in Iran was 1.2 cases per 100'000 population in 2009 [27]. It is assumed that one of the important reasons behind the resurgence of pertussis is dissimilarity between the vaccine strains and the circulating strains that has led to adaptation of the bacterium in the host [28]. Polymorphism has been thought to be the main strategy of the bacterium to escape from the immune system while the high number of variants indicate that it is beneficial for the bacterium to modulate the expression of its pertussis toxin to suppress the host immune reactions [28].

There has been a great amount of genetic variation reported in the pertussis toxin promoter region and to this date, 14 different alleles of ptxP have been found [30]. BvgA is a global regulator of $B$. pertussis virulence genes and the cooperative binding of BvgA to ptxP is required for efficient transcription of PTX. Polymorphism has been found to be restricted to the DNA region which is implicated in binding of RNA polymerase and BvgA [29].
Strains with a novel allele for the Ptx promoter (ptxP3) emerged in the 1990s, replacing the resident ptxP1 strains [30]. The variation in PTX has been shown to affect the vaccine efficacy in a mouse model [31]. A comparison of pertussis toxin production of ptxP1 and ptxP3 strains has shown that ptxP3 strains produce pertussis toxin 1.62 times more than ptxP1. It seems that the ptxP3 strains have probably been expanded globally in recent years [32]. The variation in ptx promoter has been related to increase in the colonization in a mouse model [33]. A role for vaccination in driving shifts in B. pertussis populations has also been supported by recent genomic data [18]. The ptxP3 strains have been found in 11 countries, representing 4 continents, namely Asia, Europe, as well as North and South Americas [18]. The frequency of ptxP in different countries in 1990-2000 has been shown in Table 3.

Our study is the first research about polymorphism of ptxP of B. pertussis isolated strains in Iran.

The pertussis vaccination program in Iran contains three doses of a wP vaccine. The first dose is administered at the age of 2 months, the second at 4 months and the last is administered at 6 months. Only two booster doses are administered at 18 month and 6 years. The wP is the only type of pertussis vaccine that is currently used in Iran. Despite the high coverage of the vaccine in Iran, the resurgence of Pertussis has been reported in recent years [34]. Whooping cough is mainly diagnosed in Iran among infants less than 18 months that do not complete their vaccination program (Table 2). We have observed an increase in positive cases of the disease in recent years, especially among infants under 18 months of age. It seems that the decline in the efficacy of the vaccines due to the antigenic shifts remain as an important reason for the resurgence of pertussis in vaccinated populations. WHO has suggested the re-arrangement of the vaccine strains over the years; therefore, similar studies must include periods of more than one year [35]. 
Table 3. Frequency of ptxP in different countires during 1990-2000 [18].

\begin{tabular}{|c|c|c|c|c|c|c|c|c|}
\hline \multirow[t]{3}{*}{ ptxp allele } & \multicolumn{8}{|c|}{ Country \& period } \\
\hline & \multicolumn{2}{|c|}{ Netherlands } & \multicolumn{2}{|c|}{ United States } & \multirow{2}{*}{$\begin{array}{c}\text { Australia } \\
1990-2000\end{array}$} & \multirow{2}{*}{$\begin{array}{c}\text { Finland } \\
1990-2000\end{array}$} & \multirow{2}{*}{$\begin{array}{c}\text { Italy } \\
1990-2000\end{array}$} & \multirow{2}{*}{$\begin{array}{c}\text { Japan } \\
1990-2000\end{array}$} \\
\hline & Prevacc & $1990-2000$ & Prevacc & $1990-2000$ & & & & \\
\hline$p t \times P 1$ & $50 \%(10)$ & $53 \%(178)$ & $43 \%(3)$ & $30 \%(3)$ & $90 \%(9)$ & $98 \%(48)$ & $91 \%(10)$ & $81 \%(13)$ \\
\hline$p t \times P 2$ & $45 \%(9)$ & $0.003 \%(1)$ & $29 \%(2)$ & & & & & \\
\hline$p t \times p 3$ & & $46 \%(153)$ & & $70 \%(7)$ & $10 \%(1)$ & $2 \%(1)$ & & $13 \%(2)$ \\
\hline$p t x p 4$ & $5 \%(1)$ & & $29 \%(2)$ & & & & & \\
\hline$p t \times p 5$ & & & & & & & $9 \%(1)$ & \\
\hline ptxp7 & & $0.003 \%(1)$ & & & & & & \\
\hline ptxp8 & & & & & & & & $6 \%(1)$ \\
\hline
\end{tabular}

\section{ACKNOWLEDGMENT}

We would like to show our gratitude to all staff in Department of Bacteriology and Microbiology Research Center, Pasteur Institute of Iran for sharing their wisdom with us during the course of this research.

\section{CONFLICT OF INTEREST}

The authors declare that they have no conflict of interest.

\section{REFERENCES}

1. Versteegh FG, Schellekens JF, Fleer A, Roord JJ. Pertussis: a concise historical review including diagnosis, incidence, clinical manifestations and the role of treatment and vaccination in management. Reviews in medical microbiology. 2005;16(3):79-89.

2.http://apps.who.int/iris/bitstream/10665/127891/1/WHO IVB 14.03 eng. pdf Af.

3.http://www.cdc.gov/pertussis/clinical/diagnostic-testing/specimencollection.html Af.

4. Mooi FR, He Q, Guiso N. Phylogeny, evolution, and epidemiology of Bordetellae. Bordetella molecular microbiology. 2007:17-45.

5 . Henderson IR, Nataro JP. Virulence functions of autotransporter proteins. Infection and immunity. 2001;69(3):1231-43.

6. Pillsbury A, Quinn HE, McIntyre PB, Bertilone C, Wallace T, Selvey LA et al. Epidemiology of pertussis in Australia. Communicable Diseases Intelligence. 2014;38(1):E99-E104.

7. Mooi FR, Van Loo I, King AJ. Adaptation of Bordetella pertussis to vaccination: a cause for its reemergence? Emerging infectious diseases. 2001;7(3 Suppl):526.

8. Zepp F, Heininger U, Mertsola J, Bernatowska E, Guiso N, Roord J et al. Rationale for pertussis booster vaccination throughout life in Europe. The Lancet infectious diseases. 2011;11(7):557-70.

9. Clements C, Griffiths E. The global impact of vaccines containing aluminium adjuvants. Vaccine. 2002;20:S24-S33.

10. Wendelboe AM, Van Rie A, Salmaso S, Englund JA. Duration of immunity against pertussis after natural infection or vaccination. The Pediatric infectious disease journal. 2005;24(5):S58-S61

11. Nazari F, Alé-Agha S, Mahinpour M, Mirchamsy H. Mass immunity against diphtheria and tetanus in some urban and rural areas in Iran. Arch Inst Razi. 1973;25:49-55.

12. Güriș D, Strebel PM, Bardenheier B, Brennan M, Tachdjian R, Finch E et al. Changing epidemiology of pertussis in the United States: increasing reported incidence among adolescents and adults, 1990-1996. Clinical Infectious Diseases. 1999;28(6):1230-7.

13. He Q, Viljanen MK, Nikkari S, Lyytikainen R, Mertsola J. Outcomes of Bordetella pertussis infection in different age groups of an immunized population. Journal of Infectious Diseases. 1994;170(4):873-7.

14. van Gent M, Bart MJ, van der Heide HG, Heuvelman KJ, Mooi FR. Small mutations in Bordetella pertussis are associated with selective sweeps. 2012.
15. Bartoloni A, Pizza M, Bigio M, Nucci D, Ashworth L, Irons L et al. Mapping of a protective epitope of pertussis toxin by in vitro refolding of recombinant fragments. Nature Biotechnology. 1988;6(6):709-12.

16. Decker KB, James TD, Stibitz S, Hinton DM. The Bordetella pertussis model of exquisite gene control by the global transcription factor BvgA. Microbiology. 2012;158(Pt 7):1665-76.

17. Advani A, Gustafsson L, Åhrén C, Mooi FR, Hallander HO. Appearance of Fim 3 and ptxP3-Bordetella pertussis strains, in two regions of Sweden with different vaccination programs. Vaccine. 2011;29(18):3438-42.

18. Mooi FR, van Loo IH, Van Gent M, He Q, Bart MJ, Heuvelman KJ et al. Bordetella pertussis strains with increased toxin production associated with pertussis resurgence. Emerging infectious diseases. 2009;15(8):1206.

19. De Greeff SC, De Melker HE, van Gageldonk P, Schellekens J, Van der Klis F, Mollema L et al. Seroprevalence of pertussis in The Netherlands: evidence for increased circulation of Bordetella pertussis. PLoS One. 2010;5(12):e14183.

20. Cassiday P, Sanden G, Heuvelman K, Mooi F, Bisgard KM, Popovic T. Polymorphism in Bordetella pertussis pertactin and pertussis toxin virulence factors in the United States, 1935-1999. Journal of Infectious Diseases. 2000;182(5):1402-8.

21. Mooi FR, Van Oirschot H, Heuvelman K, van der Heide HG, Gaastra W, Willems RJ. Polymorphism in the Bordetella pertussis Virulence Factors P. 69/Pertactin and Pertussis Toxin in The Netherlands: Temporal Trends and Evidence for Vaccine-Driven Evolution. Infection and immunity. 1998;66(2):670-5.

22. Gerlach G, von Wintzingerode F, Middendorf B, Gross R. Evolutionary trends in the genus Bordetella. Microbes and infection. 2001;3(1):61-72.

23. Nikbin VS, Shahcheraghi F, Lotfi MN, Zahraei SM, Parzadeh M. Comparison of culture and real-time PCR for detection of Bordetella pertussis isolated from patients in Iran. Iranian journal of microbiology. 2013;5(3):209.

24. Crowcroft N, Stein C, Duclos P, Birmingham M. How best to estimate the global burden of pertussis? The Lancet infectious diseases. 2003;3(7):413-8.

25. Berbers GA, de Greeff SC, Mooi FR. Improving pertussis vaccination. Human vaccines. 2009;5(7):497-503.

26. http://WHO/IVB//04/14. Af.

27. http://www.cdc.gov/pertussis/outbreaks/trends.html Af.

28. Mooi FR. Bordetella pertussis and vaccination: the persistence of a genetically monomorphic pathogen. Infection, Genetics and Evolution. 2010;10(1):36-49.

29. Locht C. Bordetella: molecular microbiology. Horizon Scientific Press; 2007.

30. Octavia S, Maharjan RP, Sintchenko V, Stevenson G, Reeves PR, Gilbert GL et al. Insight into evolution of Bordetella pertussis from comparative genomic analysis: evidence of vaccine-driven selection. Molecular biology and evolution. 2011;28(1):707-15.

31. Komatsu E, Yamaguchi F, Abe A, Weiss AA, Watanabe M. Synergic effect of genotype changes in pertussis toxin and pertactin on adaptation to an acellular pertussis vaccine in the murine intranasal challenge model. Clinical and Vaccine Immunology. 2010;17(5):807-12.

32. van Gent M, Bart MJ, van der Heide HG, Heuvelman KJ, Kallonen T, He Q et al. SNP-based typing: a useful tool to study Bordetella pertussis populations. 2011.

33. Bart MJ, van Gent M, van der Heide HG, Boekhorst J, Hermans P, 
Parkhill $\mathrm{J}$ et al. Comparative genomics of prevaccination and modern Bordetella pertussis strains. BMC genomics. 2010;11(1):627.

34. Ghanaie RM, Karimi A, Sadeghi H, Esteghamti A, Falah F, Armin S et

al. Sensitivity and specificity of the World Health Organization pertussis clinical case definition. International Journal of Infectious Diseases. 2010;14(12):e1072-e5.

35. Publication W. Pertussis vaccines: WHO position paperRecommendations. Vaccine. 2011;29(13):2355-6. 